\title{
Current approaches and future developments in automatic tachycardia detection and diagnosis
}

\author{
P S Astridge, G C Kaye, E J Perrins
}

Implantable automatic cardioverter/defibrillators are now commonly used to treat malignant ventricular arrhythmias. ${ }^{1}$ Early models simply detected a rapid heart rate and delivered a defibrillation shock, but more modern devices can deliver treatment as a tiered strategy of antitachycardia pacing, synchronised low energy cardioversion, and high energy defibrillation. ${ }^{23}$ Accurate detection and diagnosis of arrhythmias is essential to avoid inappropriate intervention: unnecessary shocks cause battery depletion, increase patient morbidity, and may cause degeneration from a stable to a poorly tolerated rhythm. ${ }^{45}$ An ideal system should treat haemodynamically unstable arrhythmias with a defibrillation shock, attempt pacing termination of stable ventricular tachycardia, and remain silent during stable atrial arrhythmias. Atrial fibrillation is common in patients with ventricular arrhythmias, ${ }^{6}$ and a rapid ventricular response may suggest a ventricular arrhythmia to an unsophisticated detection system. No currently available automatic detection system can reliably distinguish these situations.

Automatic tachycardia recognition by implanted antitachycardia devices depends on correct sensing and interpretation of physiological changes. Ideally, the sensed variable should alter rapidly with changes in rhythm and recover promptly after normal rhythm is restored. We describe the currently available methods for automatic tachycardia recognition and review future trends.

The table shows the variables currently proposed for automatic tachycardia detection. Many are under preliminary investigation only, and few will achieve practical usage in implantable devices.

Electrical sensing in the right ventricle The simplest method of tachycardia detection is merely to sense an increase in heart rate manifested by a series of short $R R$ intervals. This technique does not discriminate between sinus and atrial tachycardias and ventricular arrhythmias, ${ }^{78}$ and various refinements have been added to increase specificity. The onset of arrhythmia is usually more abrupt than that of sinus tachycardia and this may be used to aid detection of a pathological tachycardia. ${ }^{9}$ However, when the sinus response to sudden maximal effort is compared with the onset of ventricular tachycardia, there may be overlap in individual subjects. ${ }^{10}$ Rate stability has been used as an additional marker of a sustained arrhythmia, but may also be unreliable. ${ }^{11}$ Systems employing rate criteria show longterm reliability and require relatively little computing power but they are not diagnostic and offer no information about haemodynamic state.

ANALYSIS OF THE VENTRICULAR ELECTROGRAM The ventricular endocardial electrogram recorded from the right ventricular apex represents the majority of ventricular activation. ${ }^{12}$ Unipolar and bipolar sensing seem equally successful experimentally ${ }^{13}$ though with unipolar sensing there is a greater risk of inappropriate sensing of extraneous electrical noise. Simple comparison of electrogram amplitude allows discrimination between sinus rhythm and ventricular fibrillation ${ }^{14}$ but neither the peak amplitude nor the maximum slew rate reliably detect ventricular tachycardia. ${ }^{15}$

\section{PROBABILITY DENSITY FUNCTION}

Probability density function was used in the earliest implantable defibrillators ${ }^{16}$ : the function calculates the time spent by the sensed electrogram close to the baseline. In organised rhythms, electrogram analysis results in a well defined peak representing the baseline. In ventricular fibrillation, however, there will appear a random scatter of points, with no peak around the baseline amplitude. ${ }^{16}$ If used in isolation, this sensing system is independent of rate, and will only diagnose arrhythmias similar in configuration to ventricular fibrillation.

\section{Template matching}

Template matching involves comparison of electrogram configuration during arrhythmias with a standard template derived during sinus rhythm.

Correlation waveform analysis calculates from beat to beat the mathematical relation between a normal template and the waveform under analysis. ${ }^{1718}$ Such a system is independent of amplitude and baseline variation, but may be confounded by the development of paroxysmal bundle branch block. ${ }^{19}$ Specificity for uniform ventricular tachycardia can be increased by comparison of the suspect wave- 
Variables used for automatic tachycardia recognition

\begin{tabular}{l}
\hline Electrical sensing \\
Rate criteria: \\
Heart rate \\
Sustained high rate \\
Rate stability \\
Rate of onset \\
Activation sequence \\
Dual chamber sensing \\
Electrogram configuration: \\
Correlation waveform \\
analysis \\
Area of difference \\
analysis \\
Gradient pattern \\
detection \\
Frequency domain \\
analysis \\
Haemodynamic sensing \\
Right ventricular pressure: \\
Absolute \\
Mean \\
Pulse pressure \\
dP/dt \\
Right atrial pressure: \\
Mean \\
Waveform \\
Right ventricular \\
volumetry \\
Mixed venous oxygen \\
saturation \\
\hline
\end{tabular}

form and a template derived during previous episodes of induced arrhythmia. ${ }^{20}$ Reliability is not affected by filtering at typical levels for an implantable device. ${ }^{21}$ The "bin area method" reduces the complexity of analysis because it compares corresponding areas or "bins" in the template and the waveform under analysis by a simple error measure, and requires a fraction of the calculations. ${ }^{22}$ Data compression before correlation analysis reduces computational demands while still reliably detecting ventricular tachycardia. ${ }^{23}$

"Area of difference" analysis assesses the difference between templates of sinus rhythm and ventricular arrhythmias by totalling the absolute differences between individual sample points on the waveform under investigation and the normal template. ${ }^{15} 18$ The method is relatively efficient in terms of computing capacity required, but is affected by signal amplitude and baseline fluctuations. Critical statistical comparison suggests that algorithms using correlation waveform and area of difference analysis are similarly adept in distinguishing ventricular tachycardia from sinus rhythm or atrial fibrillation. ${ }^{24}$

So far the computing demands of rapid analogue-digital conversion and filtering for these complicated algorithms have prevented practical application. There may be additional problems affecting a chronically implanted device; animal work has demonstrated decreases of up to $18 \%$ in electrogram voltages on exercise, ${ }^{25}$ and use of chronic leads may be associated with a reduction in amplitude and slew rate of the recorded signal. ${ }^{26} 27$

Gradient pattern detection involves calculation of a first time derivative of the endocardial electrogram, in which the amplitude is proportional to the rate of change of the original electrogram. Abnormal rhythms and left bundle branch block may be reliably distinguished from normal sinus rhythm and the results seem unaffected by changes in respiration and in posture of haemodynamically stable patients. ${ }^{28}$ The system has also been shown to function accurately for the identification of a single arrhythmia configuration in real time, ${ }^{29}$ and, when a microprocessor of similar power to implantable pacemaker processors is used, can distinguish multiple arrhythmias to which the computer had previously been exposed, provided the configurations are not too similar. ${ }^{30}$ However, the numbers studied have been small and further research is needed to assess the longer term prospects for this method.

\section{Frequency domain analysis}

Comparison of frequency spectra of cardiac electrograms for the detection and diagnosis of arrhythmias has been relatively disappointing. Fast Fourier transformation is used to convert the raw signal to a plot of voltage versus frequency. This requires a large computing capacity. Appropriate filtering can highlight differences between normal and pathological electrograms. ${ }^{31} 32$ Significant differences between the centre frequency of spectra derived during sinus rhythm and ven- tricular tachycardia have been demonstrated, ${ }^{33}$ and fibrillatory and non-fibrillatory rhythms may be discriminated. ${ }^{34}$ However, in a study of open chest dogs there was overlap between values for sinus rhythm and ventricular beats in almost half the animals studied. $^{21}$

\section{ATRIAL ELECTRICAL SENSING}

Sensing the relation between atrial and ventricular activation may aid tachycardia diagnosis. However, sensing of ventricular far-field signals may obscure a low amplitude atrial signal, as in atrial fibrillation, ${ }^{35}$ and rhythms involving a 1:1 atrioventricular relation (including atrioventricular reentry and atrioventricular nodal tachycardias and ventricular tachycardia with 1:1 retrograde conduction) remain difficult to discriminate. It is possible to distinguish between anterograde and retrograde atrial depolarisation ${ }^{36} 37$ and between atrial fibrillation and regular atrial rhythms both by atrial rate calculation and amplitude distribution and probability density function generation. ${ }^{38} 39$

Simple ventricular rate criteria in combination with atrial sensing might identify ventricular tachycardia correctly in almost every patient. ${ }^{40}$ An experimental algorithm, which may be suitable for use in implantable devices, incorporates dual chamber sensing and rate criteria and was capable of discriminating atrial intrinsic and reentry tachyarrhythmias and ventricular tachycardia with and without 1:1 retrograde conduction. ${ }^{41}$ An attempt to discriminate sinus tachycardia from paroxysmal tachycardias with a 1:1 atrioventricular relation by introducing a late diastolic atrial extrasystole is effective in the laboratory: in sinus rhythm the subsequent ventricular depolarisation will be correspondingly premature, but in paroxysmal tachyarrhythmias the ventricular regularity will not be disturbed. ${ }^{41}{ }^{42}$ Clearly, the arrhythmia must be absolutely regular for this technique to work.

\section{Activation sequence}

The different sequence of myocardial depolarisation during sinus rhythm and ventricular tachycardia may be detected by multiple endocardial electrodes ${ }^{43}$ and the addition of an atrial lead may allow differentiation of sinus and supraventricular tachycardias. ${ }^{44}$ The number of electrodes required may be reduced by use of an electrode in the coronary sinus. ${ }^{45}$ It is not possible to differentiate between rhythms with 1:1 atrioventricular relation on timing alone.

\section{Haemodynamic sensing}

While the electrical sensing algorithms described are effective in detecting a pathological arrhythmia they give no information on haemodynamic instability. In many arrhythmias rapidity of heart rate is not the sole determinant of cardiovascular instability and there is no correlation between electrogram configuration and hypotension. ${ }^{46}$ 
Therefore, sensing of a haemodynamic variable, in combination with electrical events, is desirable. Because of the thromboembolic risk associated with chronic instrumentation of the left heart, studies have concentrated on the response to arrhythmias in the right heart.

\section{PHYSIOLOGICAL RESPONSES TO TACHYCARDIA}

In a thorough evaluation of the haemodynamic consequences of arrhythmias, Nakano showed that both atrial and ventricular tachycardias are associated with a decrease in mean arterial pressure, stroke volume, and cardiac output, and consequently an increase in atrial pressure bilaterally. ${ }^{47}$ The interatrial pressure gradient increases in proportion to heart rate acceleration. Increased pulmonary artery pressure and total pulmonary resistance may largely be due to the increases in left atrial pressure. After onset of tachycardia there is partial haemodynamic recovery and increased myocardial contractility caused by catecholamine activity. ${ }^{47}$ The magnitude of haemodynamic change is greater for ventricular arrhythmias because of abnormal patterns of ventricular contraction and periodic mitral regurgitation, caused by atrioventricular dissociation, that are associated with variable ventricular systolic and end diastolic pressures. ${ }^{47}$ However, echocardiography during arrhythmias has failed to demonstrate an increased incidence or severity of mitral regurgitation during stable ventricular tachycardia in humans, and impaired cardiac performance was believed to reflect reduced ventricular diastolic filling. ${ }^{48}$ Large atrial waves during arrhythmias may also represent cannon waves as the atria contract against closed atrioventricular valves..$^{49}$

In sinus tachycardia in response to exercise, mixed venous oxygen saturation falls promptly, in line with the increase in heart rate, with changes achieved in less than 10 seconds. Stroke volume also responds quickly to exercise but does not reflect the level of exertion. Maximal right ventricular pressure and $\mathrm{dP} / \mathrm{dt}$ and mean right atrial pressure increase rapidly (over 10 seconds) with exercise, and the changes observed correlate well with workload..$^{50}$

RIGHT VENTRICULAR PRESSURE MEASUREMENT Right ventricular pressure was sensed by the first laboratory model for the implantable defibrillator. Abolition of the phasic nature of this pressure triggered charging of the capacitor discharge circuit. ${ }^{51}$ During rapid right ventricular pacing to simulate ventricular tachycardia, mean right ventricular pressure showed a significant (mean increase $45 \%$ ) and sustained increase in dogs. ${ }^{52}$ However, in humans rapid ventricular pacing at a comparable rate did not cause significant fluctuation in right ventricular pressure. ${ }^{53}$ Conversely, a rapid fall in right ventricular systolic and pulse pressures at the onset of ventricular arrhythmias has also been demonstrated. ${ }^{54}$ Changes in right ventricular pressure occurred within two seconds of tachycardia onset. By estimating the ratio of right ventric- ular pulse pressure during tachycardia and at baseline, it was possible to discriminate between stable and unstable ventricular tachycardia, though in stable cases drift back to baseline over 30 seconds was noted. Evidence of some correlation between the fall in right ventricular pulse pressure and the associated fall in systemic arterial pressure has been noted, but with poor correlation with tachycardia cycle length. ${ }^{55}$ Because there is large intra-group variability changes in right ventricular pulse pressure do not allow sinus tachycardia to be distinguished from haemodynamically stable or unstable ventricular or supraventricular tachycardia. ${ }^{56}$ Changes in $\mathrm{dP} / \mathrm{dt}$ distinguished between unstable ventricular tachycardia and sinus tachycardia, but again there was extensive overlap between arrhythmia groups. ${ }^{56}$ An algorithm based on time intervals derived from dynamic right ventricular pressures may help to identify arrhythmias, but this was derived from a limited number of animal experiments in which pacing was used to simulate ventricular arrhythmias. ${ }^{57}$

\section{Right ventricular volumetry}

Right ventricular volume may be estimated continuously from changes in intracardiac impedance. ${ }^{58}$ This involves passing a constant subthreshold alternating current across the heart, between an intracardiac bipole or between intracardiac and remote surface electrodes, and measuring the varying voltage generated by this current as the intracardiac impedance changes. Errors may arise because of electrode motion and sensing of myopotentials. There is a practical method to reduce battery drain that uses a standard pacing lead, and the technique has been proposed for use in rate adaptive pacing. ${ }^{59}$ Stroke volume may be more directly measured by a Doppler sensor, suitable for chronic implantation, situated in the superior vena cava and orientated towards the ascending aorta. ${ }^{60}$ This approach is in the early stages of development but is promising.

During tachyarrhythmias cardiac output is reduced by reduced ventricular filling and systolic emptying. Right ventricular impedance during unstable ventricular tachycardia shows a significant reduction to less than $30 \%$ of that during sinus rhythm, though the changes during stable ventricular tachycardia are less pronounced. ${ }^{61}$ The variability in transcardiac impedance measured between epicardial defibrillator patches in dogs proved to be a reliable detector of ventricular fibrillation. ${ }^{62}$ Such a system removes the need for additional implanted sensors and little extra circuitry is required, but it cannot detect other arrhythmias and is unlikely to be of clinical value with increasing use of transvenous defibrillator lead systems.

\section{Right atrial pressure monitoring}

Right atrial pressure has been recommended as a physiological variable that varies consistently with changes in myocardial performance. ${ }^{63}$ Though pressures are small $(-1$ to 
$+2 \mathrm{~mm} \mathrm{Hg}$ at rest, rising to $+5 \mathrm{~mm} \mathrm{Hg}$ with exercise), significant changes have been shown during tachycardia. In anaesthetised dogs, ventricular pacing to simulate ventricular tachycardia resulted in a significant rise in mean right atrial pressure, which conversely remained stable during rapid atrial pacing imitating a supraventricular tachycardia. ${ }^{52}$ These changes have subsequently been confirmed in humans, ${ }^{53}$ and mean right atrial pressure has been shown to reach a plateau approximately 30 seconds after the onset of ventricular tachycardia, returning to the baseline level within 30 seconds of the restoration of sinus rhythm. ${ }^{64}$ This may be too slow for mean right atrial pressure estimation to be of practical use in an implantable antitachycardia device. In addition, baseline drift affecting a chronically implanted sensor becomes especially important in a low pressure chamber, and (though regular self-zeroing may partly overcome this problem) a system not reliant on absolute pressures would avoid it. The pressure waveform in the right atrium has been proposed as a variable aiding tachycardia recognition. High fidelity pressure recordings clearly demonstrate the relation between atrial and ventricular contraction and characteristic patterns have been demonstrated associated with specific atrial and ventricular arrhythmias. $^{65}$ Mean right atrial pressure showed an increase during all types of arrhythmia, regardless of haemodynamic stability, with a considerable degree of overlap between atrial and ventricular arrhythmias, though there was a preponderance of well tolerated arrhythmias in the study ${ }^{66}$ Development of a pattern-recognition algorithm and a reliable high fidelity chronic pressure sensing catheter may allow atrial pressure waveforms to become a realistic method for tachycardia diagnosis.

\section{Mixed venous oxygen saturation}

Mixed venous oxygen saturation may be measured chronically by reflectance oximetry sensors. During ventricular fibrillation in dogs, the pulsatile intra-beat variation in oxygen saturation decreased significantly and returned to baseline within 30 seconds of the restitution of sinus rhythm. ${ }^{67}$ In humans reduction in central venous oxygen saturation was greater and more rapid during exercise than at the onset of haemodynamically tolerable ventricular tachycardia. ${ }^{68}$ During unstable tachyarrhythmias increased use of peripheral oxygen results in greater desaturation, unless there is circulatory arrest and no admixture in the central veins. ${ }^{69}$ Though these changes may be helpful in the automatic determination of haemodynamic state, the considerable lag before changes occur would delay intervention.

\section{Conclusions}

Over a short period the implantable defibrillator has developed from a simple device for tachycardia detection and delivering shocks into a system in which sophisticated tiered anti-tachycardia pacing and cardioversion is now possible. To avoid spurious shocks and to optimise device performance a detection system giving increased diagnostic accuracy is desirable. This may necessitate a multisensor system, using both electrical and haemodynamic data. Dual chamber defibrillators will soon be available and may help overcome some problems in tachycardia diagnosis, particularly the difficulties in the detection of atrial fibrillation. The addition of a haemodynamic sensor might allow more appropriate application of tiered treatment. With further advances in lead and microprocessor technology a detection system capable of diagnosing all arrhythmias and determining their haemodynamic state should become a practical reality.

1 Nisam S, Mower M, Moser S. ICD clinical update: first decade, initial 10,000 patients. PACE 1991;14:255-62.

2 Ellenbogen $\mathrm{K}$, Welch $\mathrm{W}$, Luceri $\mathrm{R}$, et al. Clinical evaluation of the Guardian ATP 4210 implantable pacemaker/defibrillator: worldwide experience (abstr). $P A C E$ 1991;14:623.

3 Brachmann J, Saggau W, Schmitt C, et al. Clinical use of an antitachycardia pacemaker with integrated defibrillator (abstr). PACE 1991;14:624.

4 Manz M, Gerckens U, Luderitz B. Erroneous discharge from an implanted automatic defibrillator during supraventricular tachycardia induced ventricular fibrillation. $A m \mathcal{F}$ Cardiol 1986;57:343-4.

5 Kelly PA, Cannom DS, Garan H, et al. The automatic implantable cardioverter-defibrillator: efficacy, complications and survival in patients with malignant ventricular arrhythmias. $\mathcal{F} \mathrm{Am}$ Coll Cardiol 1988;11:1278-86.

6 Kertes P, Adams P, Higham D, Julian DG, Campbel RWF. Antecedents and characteristics of primary and RWF. Antecedents and characteristics of primary and
secondary ventricular fibrillation (abstr). Circulation 1984;70(suppl II):368.

7 Olson W, Bardy G, Mehra R, Almquist C, Biallas R. Comparison of different onset and stability algorithms for detection of spontaneous ventricular arrhythmias (abstr). PACE 1987;10:439.

8 Tomaselli G, Scheinman M, Griffin J. The utility of timing algorithms for distinguishing ventricular from supraventricular tachycardias (abstr). PACE 1987, 10:415.

9 Pless BD, Sweeney MB. Discrimination of supraventricular tachycardia from sinus tachycardia of overlapping cycle length. $P A C E$ 1984;7:1318-24.

10 Fisher JD, Goldstein M, Ostrow E, Matos J, Kim SG. Maximal rate of tachycardia development: sinus tachycardia with sudden exercise versus spontaneous ventriccardia with sudden exercise versus spo
ular tachycardia. $P A C E$ 1983;6:221-8.

11 Geibel A, Zehender M, Brugada P. Changes in cycle lengths at the onset of sustained tachycardias-importance for antitachycardia pacing. Am Heart $₹$ 1988;115: tance for

12 Nielson AP, Finke WL, Schuenemeyer T, Griffin JC. The endocardial electrogram: representative of local or distant myocardial activation? (abstr). PACE 1984;7:474.

13 Greenhut SE, DiCarlo LA, Jenkins JM, Throne RD, Winston SA. Identification of ventricular tachycardia using intracardiac electrograms: a comparison of unipolar versus bipolar waveform analysis. PACE 1991; 14:427-33.

14 Leitch JW, Yee R, Klein GJ, Jones DL, Murdock CJ. Correlation between the ventricular electrogram amplitude in sinus rhythm and ventricular fibrillation. $P A C E$ 1990;13:1105-9.

15 Langberg JJ, Gibb WJ, Auslander DM, Griffin JC. Identification of ventricular tachycardia with use of the morphology of the endocardial electrogram. Circulation 1988;77:1363-9.

16 Mirowski M, Mower MM, Reid PR, Watkins L, Langer A. The automatic implantable defibrillator: new modaliA. The automatic implantable defibrillator: new modality for treatment
$1982 ; 5: 384-401$.

17 Lin D, DiCarlo LA, Jenkins JM. Identification of ventricular tachycardia using intracavity ventricular electrograms: analysis of time and frequency domain patterns. PACE 1988;11:1592-606.

18 Tomaselli GF, Nielson AP, Finke WL, Singupta L, Clark JC, Griffin JC. Morphological differences of the endocardial electrogram in beats of sinus and ventricular origin. PACE 1988;11:254-62.

19 Throne RD, DiCarlo LA, Jenkins JM, Winston SA Paroxysmal bundle branch of supraventricular origin: a possible source of misdiagnosis in detecting ventricula tachycardia using time domain analyses of intraventricular electrograms. PACE 1990;13:453-68.

20 Throne RD, Jenkins JM, Winston SA, DiCarlo LA Confirmation of recurrent monomorphic ventricula 
tachycardia by template matching: a feasibility study [abstr]. PACE 1990;13:496.

21 Jenkins J, Feaster C, MacDonald R. Impact of filtering upon ventricular tachycardia identification by corre tion waveform analysis [abstr]. PACE 1991;14:661.

22 Throne RD, Jenkins JM, DiCarlo LA. The bin area method: a computationally efficient technique for analymethod: a computationally efficient technique for analy-
sis of ventricular and atrial intracardiac electrograms. sis of ventricular and atrial
PACE 1990;13:1286-97.

23 Steinhaus BM, Wells RT, Greenhut SE, Maas SM, Nappholtz TA, Jenkins JM. Detection of ventricula tachycardia using scanning correlation analysis. $P A C E$ 1990;13:1930-6.

24 Throne RD, Jenkins JM, DiCarlo L. Intraventricular electrogram analysis for ventricular tachycardia detection statistical validation. $P A C E$ 1990;13:1596-1601

25 Bricker JT, Ward KA, Zinner A, Gillette PC. Decrease in canine endocardial and epicardial electrogram voltages with exercise: implications for pacemaker sensing. PACE 1988;11:460-4.

26 Brouwer J, Nagelkerke D, De Jongste MJ, Boute W, Den Heijer P, Lie KI. Analysis of the morphology of the unipolar endocardial paced evoked response. $P A C E$ 1990;13:302-13.

27 Furman S, Hurzeler P, DeCaprizio V. Cardiac pacing and pacemakers. III Sensing the cardiac electrogram. Am Heart $\mathcal{f} 1977 ; 93: 794-801$

28 Davies DW, Wainwright RJ, Tooley MA, et al. Detection of pathological tachycardia by analysis of electrogram of pathological tachycardia by anal
morphology. $P A C E$ 1986;9:200-8.

29 Davies DW, Tooley MA, Cochrane T. Real time tachycardia diagnosis using morphological analysis of electrocardia diagnosis using morphological

30 Tooley MA, Davies DW, Nathan AW, Camm AJ. Recognition of multiple tachyarrhythmias by rate-independent means using a small microcomputer. $P A C E$ 1991;14:337-40.

31 Pannizzo F, Mercando AD, Fisher JD, Furman $S$ Automatic methods for detection of tachyarrhythmias by antitachycardia devices. $\mathcal{F} \mathrm{Am}$ Coll Cardiol 1988 11:308-16.

32 Pannizzo F, Furman S. Frequency spectra of ventricular tachycardia and sinus rhythm in human intracardiac electrograms-application to tachycardia detection fo cardiac pacemakers. IEEE Trans Biomed Eng 1988; 35:421-5.

33 Craelius W, Saksena S, Pantopoulos D, Hussain SM, Parsonnet V, Gielchinsky I. Frequency analysis of cardiac potentials: a new technique for ventricular tachydiac potentials: a new techm Coll Cardiol 1984;3:581.

34 Ropella K, Sahakian AV, Baerman JM, Swiryn S. The coherence spectrum-a quantitative discriminator of Circulation 1989;80:112-9.

35 Den Heijert $P$, Nagelkerke $D$, Begemann $M$, et al. Analysis of far field ventricular deflections in the righ atrial electrogram: feasibility of single lead AV interva based AAIR pacing [abstr]. PACE 1990;13:1197.

36 Wainwright $R$, Davies $W$, Tooley $M$. Ideal atrial lead positioning to detect retrograde atrial depolarisation by digitization and slope analysis of the atrial electrogram. PACE 1984;7:1152-8.

37 Throne RD, Jenkins JM, Winston SA, Finelli CJ, DiCarlo LA. Discrimination of retrograde from anterograde atrial activation using intracardiac electrogram waveform analysis. PACE 1989;12:1622-30.

38 Slocum J, Sahakian A, Swiryn S. Computer discrimination of atrial fibrillation and regular atrial rhythms from tion of atrial fibrillation and regular atrial rhythm

39 Jenkins J, Noh K, Guezennec A, Bump T, Arzbaecher R Computer algorithms for recognition of atrial fibrillation in implantable devices [abstr]. PACE 1987;10:998.

40 Schuger CD, Jackson K, Steinman RT, Lehmann MH. Atrial sensing to augment ventricular tachycardia detection by the automatic implantable cardioverter defibrillator: a utility study. $P A C E$ 1988;11:1456-64

41 Arzbaecher R, Bump T, Jenkins J, et al. Automatic tachycardia recognition. $P A C E$ 1984;7:541-7.

42 Jenkins J, Bump T, Munkenbeck F, Brown J, Arzbaecher $R$. Tachycardia detection in implantable antitachycardia devices. PACE 1984;7:1273-7.

43 Mercando AD, Furman S. Measurement of differences in timing and sequence between two ventricular electrodes as a means of tachycardia differentiation. $P A C E$ 1986;9: 1069-78.

44 Mercando $A D$, Vincenti A, Furman S, Fisher JD Tachycardia differentiation using one atrial and two ventricular electrodes [abstr]. PACE 1987;10:998.
45 Della Bella P, Brugada P, Lemery R, et al. A transcardiac lead system for identification and termination of supraventricular and ventricular tachycardia. $A m \mathcal{F}$ Cardiol 1987;60:1043-50.

46 Callans DJ, Marchlinski FE, Josephson ME. Heart rate criteria alone may fail to distinguish well and poorly tolerated ventricular tachycardia [abstr]. PACE 1991; 14:709.

47 Nakano J. Effects of atrial and ventricular tachycardias on the cardiovascular dynamics. Am $\mathcal{F}$ Physiol 1964;206: 547-55.

48 Baron SB, Huang SKS, Comess KA. Left ventricular function during stable sustained ventricular tachycardia. Chest 1989;96:275-80.

49 Goldreyer BN, Kastor JA, Kershbaum KL. The haemodynamic effects of induced supraventricular tachycardia in man. Circulation 1976;54:783-89.

50 Stangl K, Wirtzfeld A, Heinze R, Laule M, Seitz K, Gobl G. A new multisensor pacing system using stroke volume, respiratory rate, mixed venous oxygen saturation, temperature, right atrial pressure, right ventricular pressure and dP/dt. PACE 1988;11:712-24.

51 Mirowski M, Mower MM, Staewen WS. Standby automatic defibrillator. Arch Intern Med 1970;126:158-6

52 Cohen TJ, Veltri EP, Lattuca JJ, Mower MM. Haemodynamic responses to rapid pacing: a model fo
tachycardia differentiation. $P A C E$ 1988;11:1522-8.

53 Beauregard L-A, Volosin KJ, Waxman HL. Differentiation of arrhythmias by measurement of intracardiac tion of arrhythmias by measurement

54 Sharma AD, Bennett TD, Erickson $M$, Klein GJ, Yee $R$ Guiraudon $G$. Right ventricular pressure during ventricular arrhythmias in humans: potential implications for implantable antitachycardia devices. $\mathcal{F} \mathrm{Am}$ Coll Cardiol 1990;15:648-55.

55 Ellenbogen KA, Lu B, Kapadia K, Wood M, Valenta H. Usefulness of right ventricular pulse pressure as a potential sensor for haemodynamically unstable ventricular tachycardia. Am $₹$ Cardiol 1990;65:1105-11.

56 Wood M, Ellenbogen KA, Lu B, Valenta H. A prospective study of right ventricular pulse pressure and $\mathrm{dP} / \mathrm{dt}$ to discriminate induced ventricular tachycardia from supraventricular tachycardia and sinus tachycardia in man. PACE 1990;13:1148-57.

57 Bennett T, Beck R, Erickson M. Right ventricular dynamic pressure parameters for differentiation of supravenmic pressure parameters for differentiation of supraven-
tricular and ventricular rhythms [abstr]. PACE 1987; 10:415.

58 Baan J, Jong TT, Kerkhof PL, et al. Continuous stroke volume and cardiac output from intraventricular dimenvolume and cardiac output from intraventricular dimen-
sions obtained with impedance catheter. Cardiovasc Res 1981;15:328-34.

59 Chirife R. A new sensor for right ventricular and thoracic volumes using the trailing edge voltage of a pulse generator [abstr]. PACE 1991;14:659.

60 Valenta HL Jr, Wrigley RH, Ellenbogen KA, Lu B. A new haemodynamic sensor for pacemakers and defibrillators [abstr]. PACE 1991;14:659.

61 Khoury D, McAlister H, Wilkoff B, et al. Continuous right ventricular volume assessment by catheter measurement of impedance for antitachycardia system control. PACE 1989;12:1918-26.

62 Weiss SM, Einstein R, McCulloch R. Can changes in transcardiac impedance appropriately detect ventricular fibrillation? PACE 1991;14:352-57.

63 Cohen TJ. A theoretical right atrial pressure feedback heart rate control system to restore physiologic control to the rate-limited heart. $P A C E$ 1984;7:671-7.

64 Cohen TJ, Liem LB. Haemodynamic responses to induced ventricular tachyarrhythmias in man: possible applications to a haemodynamically responsive antitachycardia system [abstr]. PACE 1989;12:646.

65 Kaye GC, Perrins EJ. The recognition of tachyarrhythmias based upon changes in the right atrial pressure waveform [abstr]. PACE 1990;13:496.

66 Kaye GC, Astridge PS, Perrins EJ. Tachycardia recognition and diagnosis from changes in right atrial pressure waveform - a feasibility study. $P A C E$ 1991;14: 1384-92.

67 Erickson MK, Cheng F, Bennett TD. Behaviour of mixed venous oxygen saturation during ventricular fibrillation in dogs [abstr]. PACE 1991;14:708.

68 Faerestrand S, Ohm O-J. Sustained ventricular tachycardia and exercise induced tachycardia: haemodynamic differences [abstr]. PACE 1991;14:709.

69 Cohen TJ, Liem LB. Mixed venous oxygen saturation for differentiating stable from unstable tachycardias. $\mathrm{Am}$ Heart $\mathcal{f} 1991 ; 122: 733-40$. 